\title{
Mood disorders in women with premature ovarian insufficiency
}

\author{
Radosław Słopień \\ Department of Gynaecological Endocrinology, Poznan University of Medical Sciences, Poznan, Poland
}

\begin{abstract}
Premature ovarian insufficiency (POI) and related oestrogen deficiency is a cause of many psychological symptoms, including: depression, psychological tension, anxiety, mood lability, loss of libido, feelings of loss of femininity and attractiveness, and decreased self- and sexual esteem. Distress related to POI diagnosis may be even more confusing when $\mathrm{POI}$ is diagnosed in relation to serious health complications such as cancer, due to surgery or chemotherapy. Every case of POI requires coordinated medical advice with special attendance of a gynaecological endocrinologist, psychotherapist, and sometimes even a psychiatrist.
\end{abstract}

Key words: premature ovarian insufficiency, depression, mood disorders.

\section{Introduction}

The diagnosis of premature ovarian insufficiency (POI) is related to a variety of psychological symptoms attributed to oestrogen deficiency and psychological connotations of old age and the end of sexual life. Symptoms related to oestrogen deficiency include:depression, psychological tension, anxiety, mood lability, and loss of libido. Psychological connotations of old age and the end of sexual life are related to the feelings of loss of femininity, attractiveness, and youth, leading to decreased self- and sexual esteem. Decreased selfesteem leads to loss of confidence in bodily integrity and consequent health anxiety.

The other psychological problem particularly distressing to POI patients is loss of fertility potential, which is conceptualised as an unforeseen crisis presenting a loss or challenge to a primary life goal. It is often unexplained and can last for an indeterminate time, thereby causing overwhelming distress and stretching coping mechanisms. As an ongoing stressor, infertility has been likened to the experience of chronic illness or disability. This problem can precipitate anger, guilt, anxiety, stress, and depression as well as loss of self-esteem, sexual satisfaction, and sexual spontaneity [1]. Distress related to POI diagnosis may be even more confusing when POI is diagnosed in relation to serious health complications such as cancer [2]. It has been reported that in the initial stages after diagnosis, women may experience fear, shock, disbelief, sadness, and distress [3].

Women with $\mathrm{POI}$ as a result of surgical or chemical intervention have lower self-confidence and self-concept and also more negative body image than the controls [4].

\section{Depression in premature ovarian insufficiency patients}

In a study recruiting 160 women with POI: depression was reported in $43 \%$ of them, and $26 \%$ reported that depression occurred more than five years before POI diagnosis. Of 29 commonly assessed menopausal symptoms, women reported a mean (SD) of 14.7 (7.4) symptoms; symptom scores did not substantially decrease with time after diagnosis, and the relationship with age at diagnosis was negligible. Other common symptoms included mood swings and mental fog (> 75\%); hair loss, dry eyes, cold intolerance, and joint clicking (> 50\%); tingling in limbs and low blood pressure ( 33\%); hypothyroidism (17\%); hypoglycaemia (16\%); and gluten allergies (10\%) [5].

In a study of Deeks et al. Hospital Anxiety and Depression Scale (HADS) score for depression in women with $\mathrm{POI}$ was higher than for control women. However, the mean depression score for the POI group was still within the normal range and below the cut-off score required for mild depression. The mean anxiety score for women with surgically induced POI was within the moderate anxiety range, and the mean anxiety score for women with idiopathic or chemically-induced POI was within the mild anxiety range. Post hoc analysis found that women with surgically-induced menopause were significantly more anxious than women with idiopathic POI, and both surgically-induced and chemically-induced POI women were more anxious than controls. Post hoc assessment of the Greene anxiety subscale confirmed these findings; idiopathic POI women were less anxious than surgically induced POI women, and 
both surgically-induced and chemically-induced POI women were more anxious than controls [6].

In a study by Gibson-Helm et al. depression had been diagnosed or treated in $8.7 \%$ of premenopausal women, $24 \%$ of women with idiopathic POI, and $41 \%$ of women with medically-induced POI (by oophorectomy, chemotherapy, or radiotherapy) [7]. The mean anxiety score (subscale scores of the GCS) for the idiopathic POI group did not differ significantly from that of the control group (premenopausal women). However, the mean anxiety score was higher in the medically-induced POI women compared to both the control group (premenopausal women) and the idiopathic POI group. The mean depression score for the idiopathic POI group did not differ significantly from that of the control group (premenopausal women). However, the mean depression score was higher in the medically-induced POI group compared to the control group (premenopausal group), with a trend for a higher mean depression score in the medically-induced POI group compared to the idiopathic POI group. The idiopathic POI group did not differ from the control group (premenopausal women) in the mean psychological functioning score, but the mean psychological functioning score was significantly higher in the medically-induced $\mathrm{POI}$ group compared to both the controls (premenopausal group) and the idiopathic POI group. Previous studies have reported a high prevalence of mood disorders in women with $\mathrm{POI}$, after oophorectomy $[1,8,9]$.

Adverse association between the age at menopause and the risk of subsequent depression in postmenopausal women was shown in the meta-analysis by Georgakis MK et al. [10]. This effect was retained after controlling for premenopausal depression and hormone therapy (HT) use and was enhanced among studies examining the association of age at menopause with severe depression or among women with POI. These findings indicate that a shorter exposure to endogenous oestrogens that is linked to a longer duration of oestrogen deficiency, assessed through proxy variables, increases the risk of subsequent late-life depression and emphasises the importance of the neuroprotective and anti-depressive properties of endogenous oestrogens. Early menopause due to oophorectomy increases the risk of depression later in life $[9,11]$. Regarding the potentially protective effect of oestrogen supplementation for the treatment or prevention of postmenopausal depression, oestrogens and HT - either as monotherapy or as adjunct therapy - have been reported to improve the outcome of perimenopausal depression [12-14] as opposed to the findings for depression in postmenopausal women [15], indicating a possible window of opportunity during perimenopause for the effective use of oestrogen therapy in depression [16].

In the study of Van der Stege et al. women with POI reported more complaints of anxiety, depression, soma- tisation, sensitivity, hostility, and psychological distress. The authors reported that these symptoms are related to decreased sexual life satisfaction. They had fewer sexual fantasies and masturbated less frequently. Sexual contact was associated with less sexual arousal, reduced lubrication, and increased genital pain. However, the frequency of desire to have sexual contact and the frequency of actual sexual contact with a partner did not differ between women with POI and control women. Decreased sexual life satisfaction corelated with lower levels of oestradiol, total testosterone, and androstenedione in POI patients. Higher total testosterone levels were associated with increased frequency of desire for sexual contact, and higher androstenedione levels were associated with elevated frequency of sexual contact [17].

\section{Conclusions}

Premature ovarian insufficiency is related to the symptoms of oestrogen deficiency and psychological distress due to premature aging and the end of sexual life. These symptoms include depression, psychological tension, anxiety, mood lability, loss of libido, feelings of loss of femininity, attractiveness, and youth, decreased self- and sexual esteem, loss of confidence in bodily integrity, and consequent health anxiety. Distress related to POI diagnosis may be even more confusing when POI is diagnosed in relation to serious health complications such as cancer, due to surgery or chemotherapy.

Such a case requires coordinated medical advice with special attendance of a gynaecological endocrinologist, psychotherapist, and sometimes even a psychiatrist.

\section{Disclosure}

The author reports no conflict of interest.

\section{References}

1. Liao KL, Wood N, Conway GS. Premature menopause and psychological well-being. J Psychosomat Obstet Gynecol 2000; 21: 167-174.

2. Knobf MT. "Coming to grips" with chemotherapy-induced premature menopause. Health Care Women Int 2008; 29: 384-399.

3. Pasquali EA. Premature menopause and self-concept disjunctions. A case for crisis management. J Psychosoc Nurs Ment Health Serv 2002; 40: 20-29.

4. Schover L. Sexuality and body image in younger women with breast cancer. J Natl Cancer Inst 1994; 16: 177-182.

5. Allshouse AA, Semple AL, Santoro NF. Evidence for prolonged and unique amenorrhea-related symptoms in women with premature ovarian failure/primary ovarian insufficiency. Menopause 2015; 22: 166-174.

6. Deeks AA, Gibson-Helm M, Teede H, Vincent A. Premature menopause: a comprehensive understanding of psychosocial aspects. Climacteric 2011; 14: 565-572.

7. Gibson-Helm M, Teede H, Vincent A. Symptoms, health behawior and understanding of menopause therapy in women with premature menopause. Climacteric 2014; 17: 666-673. 
8. Vincent A, Farrell E. Premature menopause. In: Current Topics in Menopause. Dvornyk V (ed.). Bentham Science Publishers, 2013: 414-441.

9. Rocca WA, Grossardt BR, Geda YE, et al. Long-term risk of depressive and anxiety symptoms after early bilateral oophorectomy. Menopause 2008; 15: 1050-1059.

10. Georgakis MK, Thomopoulos TP, Diamantaras AA, et al. Association of Age at Menopause and Duration of Reproductive Period With Depression After Menopause: A Systematic Review and Meta-analysis. JAMA Psychiatry 2016; 73: 139-149.

11. Mantani A, Yamashita H, Fujikawa T, Yamawaki S. Higher incidence of hysterectomy and oophorectomy in women suffering from clinical depression: retrospective chart review. Psychiatry Clin Neurosci 2010; 64: 95-98.

12. Zweifel JE, O'Brien WH. A meta-analysis of the effect of hormone replacement therapy upon depressed mood. Psychoneuroendocrinology 1997; 22: 189-212.

13. Soares CN, Almeida OP, Joffe H, Cohen LS. Efficacy of estradiol for the treatment of depressive disorders in perimenopausal women: a doubleblind, randomized, placebo-controlled trial. Arch Gen Psychiatry 2001; 58: 529-534.

14. Schmidt PJ, Nieman L, Danaceau MA, et al. Estrogen replacement in perimenopause-related depression: a preliminary report. Am J Obstet Gynecol 2000; 183: 414-420.

15. Morrison MF, Kallan MJ, TenHave T, et al. Lack of efficacy of estradiol for depression in postmenopausal women: a randomized, controlled trial. Biol Psychiatry 2004; 55: 406-412.

16. Soares CN. Depression in peri- and postmenopausal women: prevalence, pathophysiology and pharmacological management. Drugs Aging 2013; 30: 677-685.

17. Van der Stege JG, Groen H, van Zadelhoff SJ, et al. Decreased androgen concentrations and diminished general and sexual well-being in women with premature ovarian failure. Menopause 2008; 15: 23-31. 